\title{
Urinary Metabonomics for Diagnosis of Depression in Hepatitis B Virus-Infected Patients
}

\author{
Li-Juan Hou ${ }^{1}$; Hong-Wei Wang ${ }^{1}$; Xiao-Xia Wei ${ }^{1}$; Shu-Peng Duan ${ }^{1}$; Ya Zhuo ${ }^{1}$; Xin-Wen Song ${ }^{1}$; \\ Bao-Sheng Shen ${ }^{1, *}$ \\ ${ }^{1}$ Department of Infectious Diseases, The First Affiliated Hospital of Xinxiang Medical University, Xinxiang, China \\ ${ }^{*}$ Corresponding Author: Bao-Sheng Shen, Department of Infectious Diseases, The First Affiliated Hospital of Xinxiang Medical University, Xinxiang, China. Tel: +86-3733029485, Fax: \\ +86-3733029645, E-mail: shenbaosheng52@163.com
}

Received: January 26, 2015; Accepted: January 28, 2015

\begin{abstract}
Background: Depression is concomitantly presented in Hepatitis B Virus (HBV)-infected patients and decreases these patients' quality of life. However, there are no laboratory-based methods to objectively diagnose this disorder.

Objectives: The aim of this study was to investigate the alteration of urinary metabolites in depressed HBV-infected patients.

Patients and Methods: In this study, 81 depressed HBV-infected patients, 68 non-depressed HBV-infected patients and 64 Healthy Controls (HC) were recruited. A nuclear magnetic resonance (NMR)-based urinary metabonomic method was used to characterize the urinary metabolic profiling of depressed and non-depressed subjects.

Results: Seventeen differential urinary metabolites responsible for discriminating depressed HBV-infected patients from non-depressed HBV-infected patients and $\mathrm{HC}$ were identified. Among these metabolites, pyruvate, isobutyrate, $\mathrm{N}$-methylnicotinamide, $\alpha$-hydroxybutyrate, acetoacetate and malonate were identified as potential biomarkers for diagnosing depression in HBV-infected patients. A combined panel of these potential biomarkers could effectively discriminate depressed HBV-infected patients from non-depressed HBV-infected patients and HC, with an average accuracy of $89.6 \%$ in the training set and a predictive accuracy of $86.4 \%$ in the test set.

Conclusions: These findings suggest that NMR-based urinary metabonomics approach might be a useful tool for the clinical diagnosis of depression in HBV-infected patients and the six potential biomarkers could be helpful for developing an objective diagnostic method. Limited by the number of recruited subjects, future studies are required to validate our conclusions.
\end{abstract}

Keywords: Depression; Hepatitis B; Metabonomic

\section{Background}

Hepatitis B Virus (HBV) is one of the most common diseases in China and infects more than two billion people around the globe (1). Hepatitis B Virus is frequently accompanied by many complications, such as cirrhosis, hepatic failure and hepatocellular carcinoma (2). Among patients infected by HBV, more than 350 million develop chronic HBV infection (1). Nowadays, HBV infection remains a serious global health concern. Infection with HBV results in more than 600000 deaths per year due to associated complications (3). Moreover, HBV is responsible for more than half of the world's hepatocellular carcinoma cases, which is amongst the three major causes of death in Africa and Asia. Depression is a debilitating mood disorder that affects up to $15 \%$ of the world's population (4), and contributes to $12.3 \%$ of the global disease burden (5). This psychiatric disorder is prevalent in chronic HBV-infected patients. A previous study conducted in Iran, showed that depression was present in $30 \%$ of these patients (6). Depression in HBV-infected patients is mainly caused by physical, cognitive, functional impairments and fear of disease complications $(7,8)$. It has been reported that depression is related to patients' feeling of fatigue (9). Meanwhile, medical treatment with interferon could also lead to depression. Depression can decrease quality of life and social activities in affected patients. Reducing the patients' compliance with prolonged therapeutic regimens is one of the most important consequences of depression (10). However, the diagnosis and treatment of depression is often overlooked in most HBV-infected patients (11). Currently, due to the lack of objective diagnostic methods, the diagnosis of depression still relies on the subjective identification of symptomatic clusters. This method is always associated with a high error rate (12). That is because the clinical presentation of depression is highly heterogeneous and standardized structured diagnostic interviews cannot sufficiently characterize this heterogeneity (13). One of methods that can be used to circumvent these limitations is to identify disease-related biomarkers to develop objective diagnostic methods. Recently, researchers have increasingly used metabonomics to explore potential biomarkers for neuropsychiatric disorders (14-17). Two studies identi- 
Hou L et al.

fied some potential biomarkers for depression through profiling metabolites in urine samples from depressed patients and healthy controls $(16,17)$. However, due to varying metabolic changes during varying disease states, it is unknown whether or not these potential biomarkers could be used to diagnose depression in HBV-infected patients. Given these facts, there is an urgent need to develop objective laboratory-based diagnostic approaches for depression in HBV-infected patients. In this study, a nuclear magnetic resonance (NMR)-based metabonomic platform was applied to profile metabolites in urine samples of depressed HBV-infected patients, non-depressed HBV-infected patients and healthy controls (HC).

\section{Objectives}

The aim of this study was to firstly identify different urinary metabolites in depressed HBV-infected patients relative to non-depressed HBV-infected patients and HC using highly homogeneous training samples. Secondly, our aim was to obtain a simplified biomarker panel for diagnosis of depression in patients with HBV-infection, and to independently validate its diagnostic performance in diverse test samples.

\section{Patients and Methods}

\subsection{Subjects}

All recruited subjects signed written informed consents before sample collection. The protocols of this research were reviewed and approved by the Ethical Committee of Xinxiang Medical University. The methods were conducted according to approved guidelines and regulations. All patients with HBV-infection were recruited from the First Affiliated Hospital of Xinxiang Medical College. The candidates who met the following criteria were included in this research:

1) a diagnosis of depression according to the Structured Psychiatric Interview using the Diagnostic and Statistical Manual of Mental Disorders-IV-Text Revision (DSM-IV-TR) criteria (18).

2) 17-item Hamilton Depression Rating Scale (HDRS) score of more than 17

3) Cirrhosis patients co-infected with Hepatitis $\mathrm{C}$ or human immunodeficiency virus were excluded.

4) Subjects with other psychiatric disorders, any preexisting bodily disorders and illicit drug use were also excluded.

5) Signed written informed consent. In total, 81 depressed HBV-infected patients were recruited.

Meanwhile, 68 non-depressed HBV-infected patients that met the third and four criteria were recruited. Healthy controls (HC) were recruited from the medical examination center of the First Affiliated Hospital of Xinxiang Medical College and had no history of DSM-IV Axis I/II, neurologic or systemic medical illness. Totally, 64 $\mathrm{HC}$ were recruited.

\subsection{Sample Preparation}

Subjects' morning urine samples were obtained after overnight fasting. We used sterile cups to collect urine samples, which were then poured into sterile tube. After centrifuging at $1500 \mathrm{~g}$ for 10 minutes, the resulting supernatant was divided into equal aliquots and stored at $-80^{\circ} \mathrm{C}$.

\subsection{Nuclear Magnetic Resonance Acquisition}

Before conducting NMR analysis, several steps were completed.

- Removal of precipitate: thawing the samples and centrifuging for 10 minutes at $1500 \mathrm{~g}$

- Stabilizing of urinary $\mathrm{pH}$ : adding $100 \mu \mathrm{L}$ of phosphate buffer to $500 \mu \mathrm{L}$ of urine

- Obtaining the supernatant: centrifuging the mixture for 10 minutes at $12000 \mathrm{rpm}$ and taking $500 \mu \mathrm{L}$ of the supernatant in $5 \mathrm{~mm}$ NMR tubes

- Collection of proton spectra: using the Bruker Avance 600 spectrometer operating at a $1 \mathrm{H}$ frequency of 600.13 $\mathrm{MHz}$ and using a standard 1D pulse sequence

- Parameters settings: 64 transients, $16 \mathrm{~K}$ data points, $8000 \mathrm{~Hz}$ spectral width, 2.0 seconds relaxation delay and $0.945 \mathrm{~s}$ acquisition time

- Reform free induction decay (FID): before Fourier transformation, zero-filling and multiplying (by an exponential line-broadening function of $0.3 \mathrm{~Hz}$ ) the FID

Urine resonance assignments were conducted in accordance with previous reports and NMR databases $(19,20)$.

\subsection{Data Analysis}

Spectra were manually phased and the baseline was corrected and referenced to 3-trimethylsilyl-1-[2,2,3,3-2 $\mathrm{H} 4]$ propionate (TSP)at $\delta 0.0(16)$. Next, NMR spectra were segmented into $0.005 \mathrm{ppm}$ chemical shift "bins" between 0.5 and 9.5 ppm using the AMIX package (Bruker Biospin, Germany). The exclusion of spectral regions of water and urea resonances before analysis was to remove baseline effects of imperfect water saturation. The normalized integral values of each sample were imported to SIMCA$\mathrm{P}+12.0$ and SPSS 19.0 software to perform multivariable statistical analysis. The spectral data was scaled to unit variance. Next, a typical ten-fold cross-validated orthogonal partial least squares discriminant analysis (OPLS-DA) model was used to visualize discrimination between depressed HBV-infected patients and non-depressed HBVinfected patients (21). Parameters, including $R^{2} X, R^{2} Y$ and $\mathrm{Q}^{2} \mathrm{Y}$ were calculated and employed to assess the quality of the OPLS-DA model (22). The goodness of fit of the model was assessed by the former two parameters and the predictability of the model was assessed by the last parameter (22). Furthermore, a 199-iteration permutation test was performed to check whether the model was over-fitted. Lower values of $\mathrm{R}^{2}$ and $\mathrm{Q}^{2}$ from the permutation test than the corresponding values from the original model indicated that the model was deemed valid and not over- 
Hou L et al.

fitted (22). The differential urinary metabolites contributing to sample discrimination were selected based on a threshold of a multivariate statistical parameter (the correlation coefficient of $|r|>0.273$, equivalent to a P value of $<0.05)$ from the coefficient loading plots of the OPLS-DA model (23).

\subsection{Potential Biomarkers Identification}

When fewer metabolites were used by the clinicians to diagnose depression in HBV-infected patients, it was more feasible, economical, convenient, and acceptable by the patients. For this reason, a step-wise logistic regression analysis based on the Bayesian Information Criterion (BIC) was carried out to optimize the metabolites with $|r|>0.273$. This procedure produced the most simplified potential metabolite biomarkers panel that had good discrimination and prediction power. To assess the diagnostic performance of this panel, a receiver-operating characteristic (ROC) curve analysis was conducted to assess the ability of this panel in differentiating depressed HBV-infected patients from non-depressed HBV-infected patients and $\mathrm{HC}$ in both training and test sets.

\subsection{Statistical Analysis}

As appropriate, student's t-test, nonparametric MannWhitney U test, Chi-square test and one-way ANOVA test were performed using the SPSS software, version 19.0. Bonferroni step-down procedure was used to control the false discovery rate (24). All tests were two-sided with statistical significance set at $P$ value $<0.05$, unless otherwise stated.

\section{Results}

The overall workflow of identifying a simplified set of urinary metabolite biomarkers for depression in HBVinfected patients is summarized in Figure 1. This study recruited 81 depressed HBV-infected patients, 68 nondepressed HBV-infected patients and 64 HC. Among these subjects, 118 (55.4\%) were male. The mean age of these subjects (mean \pm SD) was $49.6 \pm 11.4$. The score of HDRS of depressed HBV-infected patients was significantly higher than that of other subjects. No significant differences in age, gender, or body mass index were found. Detailed information is stated in Table 1.

\subsection{Differential Urinary Metabolites}

The OPLS-DA model was built using the training set (38 HC, 40 non-depressed and 53 depressed HBV-infected patients) to select the differential urinary metabolites responsible for discriminating depressed HBV-infected

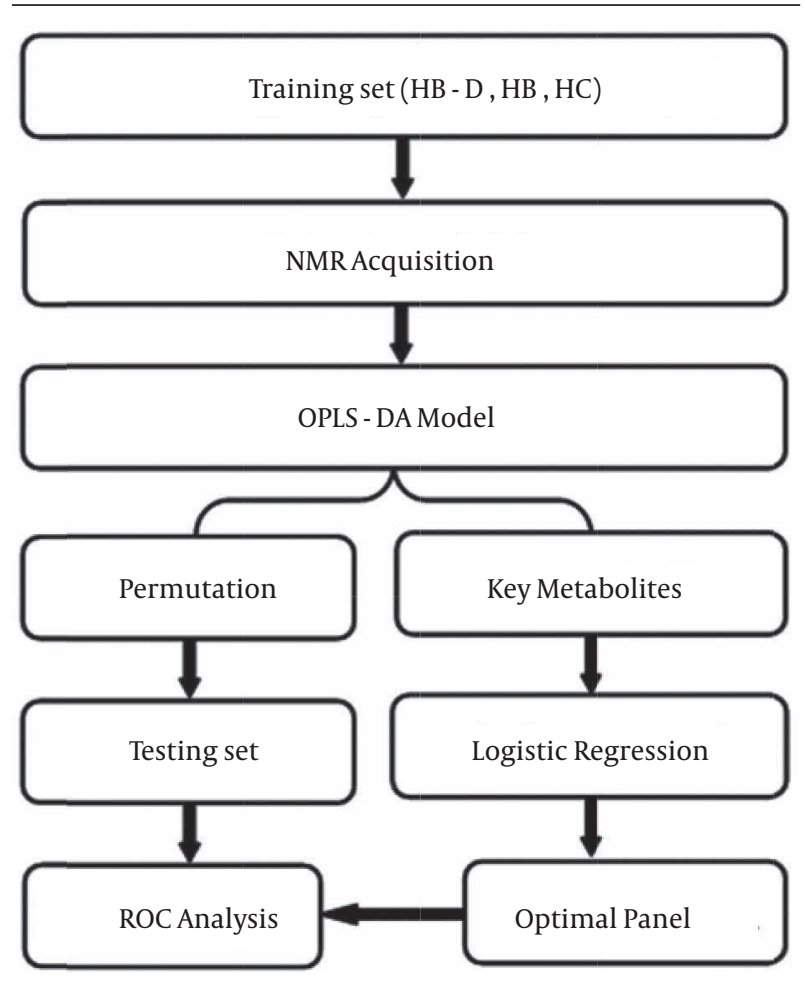

Figure 1. Overview of the Experimental Workflow

\begin{tabular}{|c|c|c|c|c|c|c|}
\hline & \multicolumn{3}{|c|}{ Training Set } & \multicolumn{3}{|c|}{ Test Set } \\
\hline & $\mathrm{HC}$ & HB & dHB & HC & HB & dHB \\
\hline Sample Size & 38 & 40 & 53 & 26 & 28 & 28 \\
\hline Age, $y$ & $49.6 \pm 11.4$ & $51.6 \pm 10.5$ & $48.7 \pm 11.2$ & $50.9 \pm 9.7$ & $49.2 \pm 10.1$ & $51.8 \pm 10.9$ \\
\hline \multicolumn{7}{|l|}{ Gender } \\
\hline Female & 17 & 17 & 18 & 10 & 12 & 11 \\
\hline Male & 21 & 23 & 25 & 16 & 16 & 17 \\
\hline HDRS & $0.4 \pm 0.5$ & $1.4 \pm 0.8$ & $23.4 \pm 4.7$ & $0.3 \pm 0.4$ & $1.9 \pm 1.1$ & $23.5 \pm 4.2$ \\
\hline BMI, $\mathrm{kg} / \mathrm{m}^{2}$ & $21.7 \pm 2.8$ & $21.4 \pm 2.5$ & $22.1 \pm 2.6$ & $22.4 \pm 2.2$ & $20.9 \pm 3.1$ & $21.5 \pm 2.7$ \\
\hline
\end{tabular}

\footnotetext{
a Abbreviations: BMI, Body Mass Index; dHB, depressed hepatitis B virus infected patients; HC, healthy controls; HB, non-depressed hepatitis B virus infected patients; HDRS, Hamilton Depression Rating Scale.
}

${ }^{\mathrm{b}}$ Data are presented as Mean \pm SD. 
patients from non-depressed HBV-infected patients and HC. The score plots of the OPLS-DA model showed that depressed HBV-infected patients were significantly different from subjects in the other two groups with little overlap (Figure $2 \mathrm{~A})$. Meanwhile, the positive values of $\mathrm{R}^{2} \mathrm{X}(61.0 \%)$, $\mathrm{R}^{2} \mathrm{Y}(64.3 \%)$ and $\mathrm{Q}^{2} \mathrm{Y}(57.8 \%)$ implied a robust metabolic difference between depressed HBV-infected patients and non-depressed subjects. Moreover, the results of permutation test demonstrated that this OPLS-DA model was valid, positive and not over-fitted (Figure 2 B). In order to independently validate the reliability of the constructed OPLS-DA model, a test set (26 HC, 28 non-depressed and 28 depressed HBV-infected patients) was used. The corresponding results showed that the OPLS-DA model could correctly predict depressed HBV-infected patients (Figure $3 \mathrm{~A}$ ), non-depressed HBV-infected patients and HC subjects
(Figure $3 \mathrm{~B}$ ). By analyzing the coefficient loading plots, 17 differential urinary metabolites $(|\mathrm{r}|>0.273)$ that contributed to the discrimination of depressed HBV-infected patients were obtained. Among these metabolites, the levels of five metabolites (acetoacetate, glycerophosphocholine, malonate, phenylacetylglycine and p-hydroxyphenylacetate) were lower in depressed HBV-infected patients, and the levels of 12 metabolites (isobutyrate, acetone, $\alpha$-hydroxybutyrate, glycolate, $\beta$-hydroxybutyrate, pyruvate, trimethylamine, dimethylglycine, $\alpha$-hydroxyisobutyrate, 3,4-dihydroxymandelate, acetamide and N-methylnicotinamide) were higher in depressed HBV-infected patients. Meanwhile, the non-parametric Mann-Whitney U test was used to confirm the metabolic changes identified by the OPLS-DA model, and the 17 metabolites still remained significantly changed (Table 2 ).
A

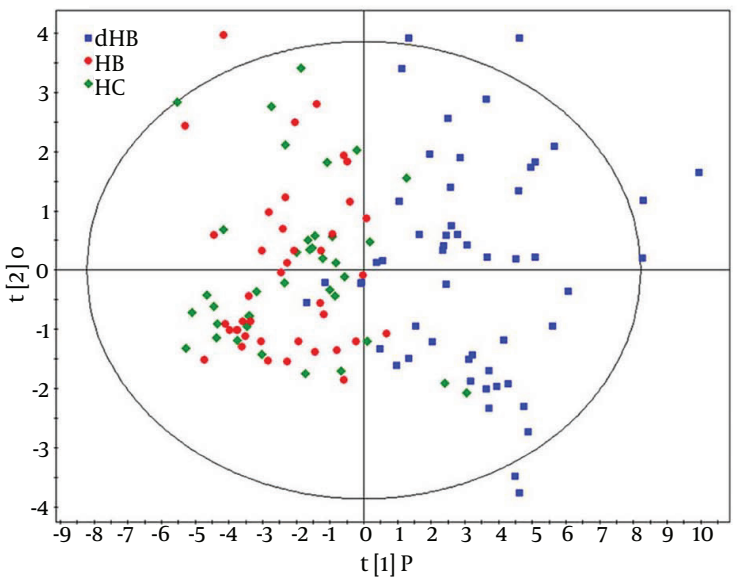

B

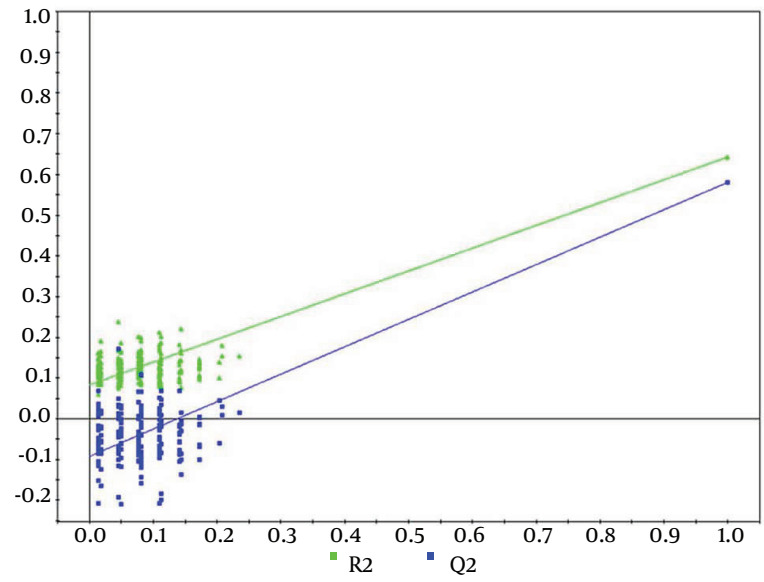

A, The score plots of the OPLS-DA model showing an obvious separation between depressed subjects (blue box, HB-D) and non-depressed subjects (red dot, HB; green diamond, HC); B, 199 permutation test.

\section{Figure 3. T-Predicted Scatter Plot from the OPLS-DA Model}

A

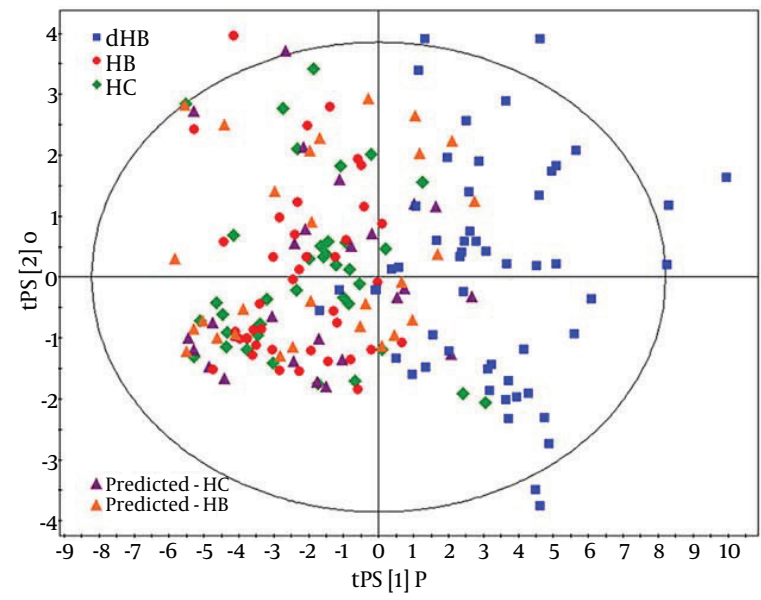

B

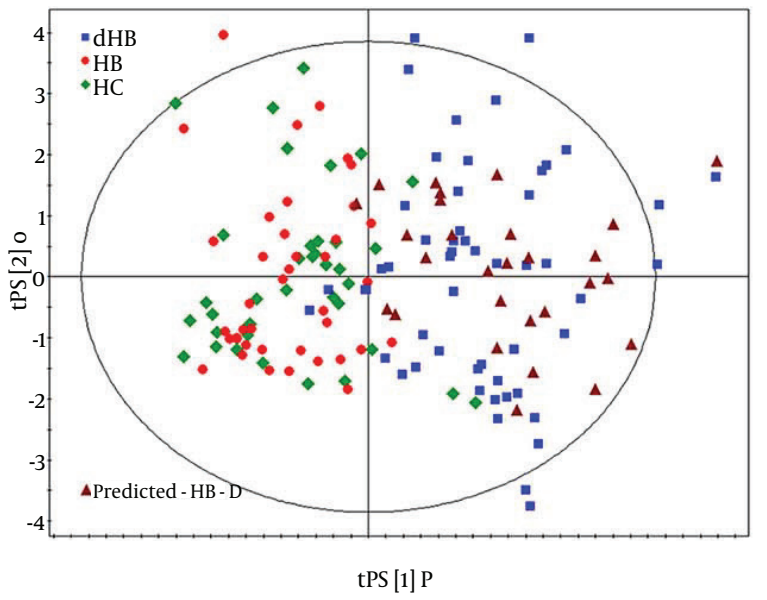


Hou L et al.

\begin{tabular}{|c|c|c|c|c|}
\hline No & Metabolite & $\mathbf{R}^{\mathrm{a}}$ & PValue $^{b}$ & Chemical Shift/ppm Multiplicity ${ }^{\mathrm{C}}$ \\
\hline 1 & 3, 4-Dihydroxymandelate & 0.520 & $1.97 \mathrm{E}-10$ & $6.87, \mathrm{~d} ; 6.92, \mathrm{~s} ; 6.98, \mathrm{~d}$ \\
\hline 2 & Acetamide & 0.392 & $4.32 \mathrm{E}-12$ & $2.00, \mathrm{~s}$ \\
\hline 3 & Acetoacetate & -0.276 & $3.62 \mathrm{E}-07$ & $2.27, \mathrm{~s} ; 3.43, \mathrm{~s}$ \\
\hline 4 & Acetone & 0.817 & $1.41 \mathrm{E}-09$ & $2.22, \mathrm{~s}$ \\
\hline 5 & Dimethylglycine & 0.432 & $1.32 \mathrm{E}-10$ & $2.78, \mathrm{~s}$ \\
\hline 6 & Glycerophosphocholine & -0.375 & $1.04 \mathrm{E}-05$ & $3.23, \mathrm{~s}$ \\
\hline 7 & Glycolate & 0.571 & $8.62 \mathrm{E}-12$ & $3.59, \mathrm{~s}$ \\
\hline 8 & Isobutyrate & 0.826 & $2.80 \mathrm{E}-12$ & $1.14, \mathrm{~d}$ \\
\hline 9 & Malonate & -0.393 & $3.95 \mathrm{E}-02$ & $3.12, \mathrm{~s}$ \\
\hline 10 & N-Methylnicotinamide & 0.386 & 2.62 E-07 & $8.21, \mathrm{~d} ; 8.90, \mathrm{~d} ; 8.97, \mathrm{~d} ; 9.29, \mathrm{~s}$ \\
\hline 11 & Phenylacetylglycine & -0.527 & 8.85 E-04 & 7.28, d; 7.36, t; 7.42, dd \\
\hline 12 & p-Hydroxyphenylacetate & -0.571 & 5.39 E-04 & $3.78, \mathrm{~s} ; 7.14, \mathrm{~d} ; 7.21, \mathrm{~d}$ \\
\hline 13 & Pyruvate & 0.491 & $6.50 \mathrm{E}-15$ & $2.38, \mathrm{~s}$ \\
\hline 14 & Trimethylamine & 0.479 & $3.10 \mathrm{E}-12$ & $2.88, \mathrm{~s}$ \\
\hline 15 & $\alpha$-Hydroxybutyrate & 0.686 & $1.21 \mathrm{E}-12$ & $1.64, \mathrm{~m} ; 1.73, \mathrm{~m} ; 3.99, \mathrm{dd}$ \\
\hline 16 & $\alpha$-Hydroxyisobutyrate & 0.404 & 3.89 E-13 & $3.69, \mathrm{~m} ; 2.65, \mathrm{~m} ; 1.11, \mathrm{~d}$ \\
\hline 17 & $\beta$-Hydroxybutyrate & 0.531 & $9.12 \mathrm{E}-07$ & $1.24, \mathrm{~d} ; 2.33, \mathrm{dd} ; 2.40, \mathrm{dd}$ \\
\hline
\end{tabular}

\subsection{Potential Metabolite Biomarkers Panel}

Under the premise of guaranteeing distinguish accuracy, the less metabolite in the diagnostic panel, the more convenient and economical in clinical use. Therefore, the 17 metabolites with $|r|>0.273$ were used as variables to conduct step-wise logistic regression analysis. The Bayesian information criterion (BIC) rule was used to determine the minimum number of metabolites that are needed to effectively separate depressed HBV-infected patients from subjects in the other two groups. This procedure yielded an optimal diagnostic panel consisting of pyruvate, isobutyrate, N-methylnicotinamide, $\alpha$-hydroxybutyrate, acetoacetate and malonate, which indicated that the six metabolites could describe the most significant deviations between depressed HBV-infected patients and subjects in the other two groups. This panel yielded an average accuracy of $89.6 \%$ in the training set and a predictive accuracy of $86.4 \%$ in the test set.

\subsection{Diagnostic Performance}

In order to quantitatively assess the diagnostic performance of this panel, a receiver-operating characteristic (ROC) curve analysis was carried out, and the area under the curve (AUC) was calculated. The results indicated that this panel could effectively discriminate the depressed HBV-infected patients in the training set with an AUC of 0.977 (95\%CI = 0.957 - 0.997) (Figure 4). Furthermore, the testing set was used to independently assess the diagnostic performance of this panel, and the value of AUC was $0.966(95 \% \mathrm{CI}=0.932-0.999)$ (Figure 4). These results showed that the diagnostic performance of this panel composed of only six metabolites and the OPLS-DA model composed of all detected metabolites were comparable.
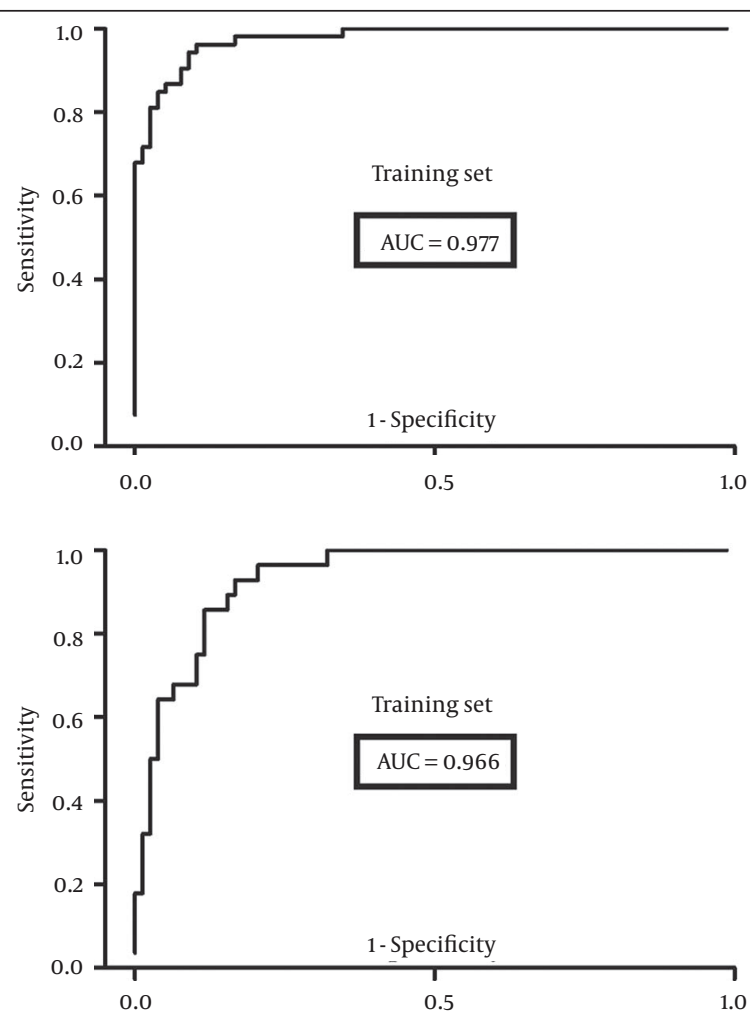

Figure 4. Receiver-Operating Characteristic Curves Analysis 


\section{Discussion}

Chronic infection of hepatitis B that causes drastic consequences such as liver dysfunction is viewed by clinicians and researchers as an important infectious disease. Depression, a psychiatric disorder, is concomitantly present in HBV-infected patients and decreases the patients' quality of life. Depression might lead to suicide, homicide or intentional transmission of infection to others. However, up to now, diagnosis of depression in HBV-infected patients has still remained subjective, and no objective laboratory based test could be used to aid clinicians with making an accurate diagnosis. In this study, a NMR-based metabonomic approach was used to identify potential diagnostic biomarkers for depressed HBV-infected patients. Consequently, a panel consisting of pyruvate, isobutyrate, $\mathrm{N}$-methylnicotinamide, $\alpha$-hydroxybutyrate, acetoacetate and malonate was identified as an effective classifier. In the training set, this panel yielded an AUC of 0.977 with sensitivity of $86.8 \%$ and specificity of $92.3 \%$. In the testing set, this panel yielded an AUC of 0.966 with sensitivity of $82.1 \%$ and specificity of $90.7 \%$. These results indicated that the diagnostic performance of these six metabolites was significantly higher than that of the currently available psychiatric interview-based methods (25). Our findings showed that urinary metabolite biomarkers might facilitate the detection of depression in HBV-infected patients, and provide candidate biomarkers for the development of objective diagnostic methods. Chronic stress might have an important role in depression development of chronic HBV-infected patients. This could be due to the increase of plasma cortisol levels and impaired serotonin uptake (26). The former was a stress-associated agent and the later was a known agent that had a role in depression. Other researchers demonstrated that chronic stress plays a critical role in the beginning and development of depression (27). They found that the plasma cortisol level of a normal person was decreased throughout the day. However, this phenomenon did not exist in depressed patients. Moreover, the level had a substantial increase in the afternoon in depressed patients. This elevation was described as a characteristic of hypercortisolism that was observed in chronic stress. A previous NMR study of 126 depressed patients and 134 healthy controls identified a potential biomarker panel for discriminating depressed patients from healthy controls (16). Two metabolites (malonate and N-methylnicotinamide) were also identified in our study. We used this panel to distinguish depressed HBV-infected patients from subjects in the other two groups. The results showed that the previous panel identified by Zheng et al. (16) could not efficiently diagnose depression in HBV-infected patients. The diagnostic accuracies were inferior to the accuracies of our panel (86.8\% and $82.1 \%$ in the training and testing set). These findings indicated that the previous panel might not be appropriate for diagnosing depressive symptoms in HBV-infected patients. This might be because meta- bonomics was applied to capture metabolic changes in disease states (28), yet different disease states might have different metabolic profiling. Therefore, the metabolic profiling of depressed HBV-infected patients in this study might be different from the metabolic profiling of depressed patients from previous work (16). There were several notable limitations in this work. First, the number of recruited subjects was relatively small. Second, whether or not this panel could be used for a wide range of patients is unknown, because all subjects were recruited from the same ethnicity and site. Third, we obtained this panel by only recruiting depressed HBV-infected patients and non-depressed subjects; therefore, whether this panel could be used to discriminate depressed HBV-infected patients from HBV-infected patients with other neuropsychiatric disorders requires further studies. Finally, any single metabonomic platform alone cannot completely cover the entire human metabonome; future studies applying other metabonomic methods are required (29). Our study provided evidence that NMR-based urinary metabonomics approach might be used as a useful tool in the clinical diagnosis of depression in HBV-infected patients. Additionally, a panel of six potential urinary metabolite biomarkers, including pyruvate, isobutyrate, $\alpha$-hydroxybutyrate, malonate, $\mathrm{N}$-methylnicotinamide and acetoacetate, was identified as an effective classifier.

\section{Acknowledgements}

We thank the doctors and nurses from the First Affiliated Hospital of Xinxiang Medical College for performing the sample collection.

\section{Authors' Contributions}

Design of the experiments: Bao-Sheng Shen, Xin-Wen Song and Li-Juan Hou. Performance of the experiments: Hong-Wei Wang and Xiao-Xia Wei. Analysis of the data: Li-Juan Hou, Shu-Peng Duan and Ya Zhuo. Provision of reagents/materials/analysis tools: Li-Juan Hou, Shu-Peng Duan and Hong-Wei Wang. Writing of the paper: BaoSheng Shen, Xin-Wen Song and Li-Juan Hou.

\section{Funding/Support}

This work was supported by a fund from the Health Department of the Henan Province (201303106) and the Education Department of the Henan Province (200733001).

\section{References}

1. Hepatitis B vaccines. Wkly Epidemiol Rec. 2009;84(40):405-19.

2. Marcellin P, Lau GK, Zeuzem S, Heathcote EJ, Pockros PJ, Reddy $\mathrm{KR}$, et al. Comparing the safety, tolerability and quality of life in patients with chronic hepatitis $B$ vs chronic hepatitis $C$ treated with peginterferon alpha-2a. Liver Int. 2008;28(4):477-85.

3. Goldstein ST, Zhou F, Hadler SC, Bell BP, Mast EE, Margolis HS. A mathematical model to estimate global hepatitis B disease burden and vaccination impact. Int J Epidemiol. 2005;34(6):1329-39.

4. Kessler RC, Berglund P, Demler O, Jin R, Koretz D, Merikangas KR, et al. The epidemiology of major depressive disorder: results 
from the National Comorbidity Survey Replication (NCS-R). JAMA. 2003;289(23):3095-105.

5. Reynolds EH. Brain and mind: a challenge for WHO. Lancet. 2003;361(9373):1924-5.

6. Ebrahimi DN, Bashashati M, Karbalaeian M, Keramati MR, Shadman YAA. Prevalence of psychiatric disorders in hepatitis B virus carriers in Iranian charity for hepatic patients support (December 2004-August 2005). Hepat Mon. 2008;8(3):201-5.

7. Gallegos-Orozco JF, Fuentes AP, Gerardo Argueta J, Perez-Pruna C, Hinojosa-Becerril C, Sixtos-Alonso MS, et al. Health-related quality of life and depression in patients with chronic hepatitis C. Arch Med Res. 2003;34(2):124-9.

8. Miotto EC, Campanholo KR, Machado MA, Benute GG, Lucia MC, Fraguas RJ, et al. Cognitive performance and mood in patients on the waiting list for liver transplantation and their relation to the model for end-stage liver disease. Arq Neuropsiquiatr. 2010;68(1):62-6.

9. McDonald J, Jayasuriya J, Bindley P, Gonsalvez C, Gluseska S. Fatigue and psychological disorders in chronic hepatitis C. J Gastroenterol Hepatol. 2002;17(2):171-6.

10. Arvand J, Shafiabadi A, Falsafinejad MR, Naderi N. Depression in patients with chronic hepatitis B: an experience on individual solution-focused therapy. Gastroenterol Hepatol Bed Bench. 2012;5(3):166-8.

11. Ozdemir S, Yalug I, Mert A, Aker T. Chronic hepatitis from a psychiatric point of view. Anatol J Psychiatry. 2008;9(4):253-60.

12. Mitchell AJ, Vaze A, Rao S. Clinical diagnosis of depression in primary care: a meta-analysis. Lancet. 2009;374(9690):609-19.

13. Chen L, Eaton WW, Gallo JJ, Nestadt G. Understanding the heterogeneity of depression through the triad of symptoms, course and risk factors: a longitudinal, population-based study. J Affect Disord. 2000;59(1):1-11.

14. Yang J, Chen T, Sun L, Zhao Z, Qi X, Zhou K, et al. Potential metabolite markers of schizophrenia. Mol Psychiatry. 2013;18(1):67-78.

15. Rhee EP, Gerszten RE. Metabolomics and cardiovascular biomarker discovery. Clin Chem. 2012;58(1):139-47.

16. Zheng P, Wang Y, Chen L, Yang D, Meng H, Zhou D, et al. Identification and validation of urinary metabolite biomarkers for major depressive disorder. Mol Cell Proteomics. 2013;12(1):207-14.

17. Chen JJ, Liu Z, Fan SH, Yang DY, Zheng P, Shao WH, et al. Combined application of NMR- and GC-MS-based metabonomics yields a superior urinary biomarker panel for bipolar disorder. Sci Rep. 2014;4:5855.
18. American Psychiatric Association.. Diagnostic and Statistical Man ual of Mental Disorders.Washington, DC: American Psychiatric Association; 2001

19. Beckwith-Hall BM, Nicholson JK, Nicholls AW, Foxall PJ, Lindon JC, Connor SC, et al. Nuclear magnetic resonance spectroscopic and principal components analysis investigations into biochemical effects of three model hepatotoxins. Chem Res Toxicol. 1998;11(4):260-72.

20. Yap IK, Angley M, Veselkov KA, Holmes E, Lindon JC, Nicholson JK. Urinary metabolic phenotyping differentiates children with autism from their unaffected siblings and age-matched controls. Proteome Res. 2010;9(6):2996-3004.

21. Domenici E, Wille DR, Tozzi F, Prokopenko I, Miller S, McKeown A, et al. Plasma protein biomarkers for depression and schizophrenia by multi analyte profiling of case-control collections. PLoS One. 2010;5(2)

22. Mahadevan S, Shah SL, Marrie TJ, Slupsky CM. Analysis of metabolomic data using support vector machines. Anal Chem. 2008;80(19):7562-70.

23. Cloarec O, Dumas ME, Trygg J, Craig A, Barton RH, Lindon JC, et al. Evaluation of the orthogonal projection on latent structure model limitations caused by chemical shift variability and improved visualization of biomarker changes in $1 \mathrm{H}$ NMR spectroscopic metabonomic studies. Anal Chem. 2005;77(2):517-26.

24. Ge Y, Sealfon SC, Speed TP. Some Step-down Procedures Controlling the False Discovery Rate under Dependence. Stat Sin. 2008;18(3):881-904.

25. Schubert DS, Burns R, Paras W, Sioson E. Increase of medical hospital length of stay by depression in stroke and amputation patients: a pilot study. Psychother Psychosom. 1992;57(1-2):61-6.

26. Alian S, Masoudzadeh A, Khoddad T, Dadashian A, Ali Mohammadpour R. Depression in hepatitis B and C, and its correlation with hepatitis drugs consumption (interfron/lamivodin/ribaverin). Iran J Psychiatry Behav Sci. 2013;7(1):24-9.

27. Tafet GE, Idoyaga-Vargas VP, Abulafia DP, Calandria JM, Roffman SS, Chiovetta A, et al. Correlation between cortisol level and serotonin uptake in patients with chronic stress and depression. Cogn Affect Behav Neurosci. 2001;1(4):388-93.

28. Kaddurah-Daouk R, Kristal BS, Weinshilboum RM. Metabolomics: a global biochemical approach to drug response and disease. Annu Rev Pharmacol Toxicol. 2008;48:653-83.

29. Dzeja P, Bouatra S, Aziat F, Mandal R, Guo AC, Wilson MR, et al. The Human Urine Metabolome. PLoS ONE. 2013;8(9). 